\title{
Transanal versus transabdominal specimen extraction in laparoscopic rectal cancer surgery: a retrospective analysis from China
}

\author{
Ruchen Wang ${ }^{1}$, Zhewei Wei ${ }^{2}$, Qilong Liư ${ }^{3}$, Wenfeng $\mathrm{Li}^{3}$, Longbin Xiao ${ }^{1}$, Fang-hai Han $\mathrm{Han}^{2}$, Shibin Yang ${ }^{3}$ \\ ${ }^{1}$ Department of Cardiothoracic Surgery, Huangpu Division of the First Affiliated Hospital, Sun Yat-sen University, Guangzhou, \\ Guangdong, China \\ 2Department of Gastrointestinal Surgery, First Affiliated Hospital, Sun Yat-sen University, Guangzhou, Guangdong, China \\ ${ }^{3}$ Department of Gastrointestinal Surgery, Huangpu Division of the First Affiliated Hospital, Sun Yat-sen University, Guangzhou, \\ Guangdong, China
}

Videosurgery Miniinv 2019; 14 (2): 203-209 DOI: https://doi.org/10.5114/wiitm.2018.79529

\begin{abstract}
Introduction: Comparison of transanal specimen extraction (TSE) and transabdominal specimen extraction (TASE) in laparoscopic rectal surgery is still sparsely reported. Trauma, pain, scarring, and bad psychological suggestion have long been considered an inevitable outcome of surgery. For laparoscopic rectal cancer surgery, whether TSE or TASE is beneficial in terms of technical platforms, indications, contraindications, technical requirements for aseptic operation, tumor-free operation, prevention and treatment of complications still has not reached a unified consensus and standards. Recently, comparison of TSE and TASE in laparoscopic rectal surgery has still been sparsely reported. Aim: In this study, we retrospectively analyzed the short-term outcomes of TSE and TASE in laparoscopic rectal surgery in a single institution in southern China.

Material and methods: Patients who underwent laparoscopic radical rectal cancer surgery using either TSE or TASE were recruited. Data, including patient demographics, perioperative and postoperative variables, were analyzed retrospectively.

Results: Sixty-seven patients were included in this study. Thirty patients underwent TSE and 37 patients underwent TASE. The two groups were similar in demographics and tumor characteristics. Postoperative complications were similar in both groups, except that wound infection was lower for the TSE group ( $p=0.122)$. The TSE group had a better cosmetic result with no abdominal incision and no differences in circumferential margins, distal resection margins or completeness of total mesorectal excision.

Conclusions: Laparoscopic TSE is recommended in the treatment of rectal cancer with similar oncologic outcomes compared with conventional TASE. It is mini-invasive surgery and has the advantage of better cosmetic results. There is a need for further randomized studies to refine the applicability of laparoscopic TSE in rectal cancer.
\end{abstract}

Key words: laparoscopic radical rectal cancer surgery, transanal specimen extraction, transabdominal specimen extraction. 


\section{Introduction}

There was no doubt that total mesorectal excision (TME) for rectal cancer had relatively low recurrence rates [1]. Over the past 2 decades, numerous prospective randomized studies have demonstrated the feasibility, safety, and advantages for laparoscopic TME for rectal cancer [2-6]. However, a 5-7 cm incision was still needed for conventional laparoscopic rectal cancer surgery and it might increase perioperative morbidity and mortality. Trauma, pain, scarring, and bad psychological suggestion have long been considered an inevitable outcome of surgery. For laparoscopic rectal cancer surgery, whether transanal specimen extraction (TSE) or transabdominal specimen extraction (TASE) is beneficial in terms of technical platforms, indications, contraindications, technical requirements for aseptic operation, tumor-free operation, prevention and treatment of complications still has not reached a unified consensus and standards. Recently, comparison of TSE and TASE in laparoscopic rectal surgery has still been sparsely reported. In this study, we retrospectively analyzed the short-term outcomes of TSE and TASE in laparoscopic rectal surgery in a single institution in southern China. The TASE surgical method: Separation of the mesentery and disconnection of the rectum; after the rectum is dissected, the proximal intestinal tube is cut off and placed in the stapler head to complete the laparoscopic wound anastomosis. TSE group: conventional abdominal surgery is the same as above. Insertion of the stapler holder: the stapler holder is connected to a guide tube, and the guide tube is placed from the anus. The guide tube is drawn from the wall of the colon wall, and the stapler holder is guided into the proximal colon by the guide tube. The self-puncture hole leads to the stapler pull rod, and the endoscope is used to suture the staple holder around the puncture hole, and the guide tube is taken out. The distal side of the intestine is turned over through the anus. Complete anastomosis: after removing the tumor and rectum according to the above requirements outside the anus, disinfect the intestine cavity to be anastomosed again with iodophor. The surgeon replaces the glove, distilled water or 5-FU into the intestinal lumen. After the rectal stump is resected, the stapler enters from the anus, and the descending colon and the rectum end are anastomosed under the laparoscopic direct view of the abdominal cavity, and the intestinal tube is relaxed without tension. The anastomosis was tested for leaks using a conventional pressurized gas injection method. Rinse and drain.

\section{Aim}

In this study, we retrospectively analyzed the short-term outcomes of TSE and TASE in laparoscopic rectal surgery in a single institution in southern China.

\section{Material and methods}

The study protocol was approved by the Ethics and Scientific Committee of our hospital. All patients and their families provided informed written consent for their information to be stored in the hospital database and used for research before surgery.

We retrospectively reviewed the medical records of patients who were diagnosed with rectal cancer by preoperative electronic endoscope colonoscopy and pathological biopsy at our department from January 2011 to September 2013. Patients who underwent laparoscopic radical sphincter-preserving rectal cancer surgery using either TSE or TASE were recruited. Taking into account the principle of surgery without tumor and sterility, we made the following provisions for the surgical indications of both: Inclusion criteria was defined as below: a) no distal metastasis; b) preoperative ultrasound endoscopic indication of $T$ stage $\leq T 3$, no sacrum and pelvic sidewall infiltration; c) preoperative pelvic MRI considerations: no pelvic lymph node metastasis. However, the main difference between the two procedures is to consider the size of the tumor and the distance from the dentate line, namely: the tumor circumference $\leq 1 / 2$ circle, the dentate line is less than $10 \mathrm{~cm}$ row TSE, the diameter of the tumor is larger than $1 / 2$ of the diameter of the intestine, TASE from the dentate line is greater than $10 \mathrm{~cm}$. Both exclusion criteria included: a) distal metastasis; b) lymph node metastasis around the abdominal aorta or pelvic iliac vessels; c) T4 stage cases; d) synchronous colorectal cancer; e) other benign or malignant tumors except adenocarcinoma; f) history of neoadjuvant therapy; g) patients who underwent simultaneous, multiorgan resections (such as liver and rectal resections). All the operations in the two groups were performed by the same senior surgeon (Fang-hai Han MD) who had performed over 200 laparoscopic radical rectal cancer procedures. Data, including patient demograph- 
ics, perioperative and postoperative details, were collected prospectively.

\section{Surgical technique}

Laparoscopic TSE and TASE surgical techniques followed the same surgical and oncologic principles: high ligation of the inferior mesenteric artery and vein to provide adequate bowel length for a tension-free anastomosis, sharp TME for mid and lower rectal cancer, preservation of the autonomic pelvic nerves and neurovascular bundle (NVB), and adequate distal and circumferential margins. The detail surgical techniques of laparoscopic TSE were well described in our early work [7]. For laparoscopic TASE, the infraumbilical Hasson incision was about $5-7 \mathrm{~cm}$ in length and protected with a small Alexis wound protector (Applied Medical, Rancho Santa Margarita, CA) for specimen extraction.

\section{Statistical analysis}

The SPSS 19.0 statistical software was used to analyze the data. Measurement data were shown as the mean \pm standard deviation $(x \pm S D)$. Analysis was performed with the 2 independent samples $t$-test, $\chi^{2}$ test or 2 independent samples tests where appropriate. In all cases, a $p$-value less than 0.05 was considered statistically significant.

\section{Results}

Sixty-seven patients were included in this study. Thirty patients underwent TSE and 37 patients underwent TASE. All patients underwent laparoscopic surgery without conversion to open surgery. There was no significant difference in gender, age, body mass index (BMI), the diameter of tumor or previous abdominal surgery between the two groups (Table I).

Intraoperative variables are summarized in Table II. The operative time in TSE and TASE surgery groups was $188.27 \pm 70.89 \mathrm{~min}$ and $190.54 \pm 65.27 \mathrm{~min}$ respectively $(p=0.010)$. With regard to the pathology results, lymph node extraction in the TSE group and in the TASE group was $14.23 \pm 7.82$ and $16.03 \pm 10.10$ respectively, and there was no significant difference between the two groups $(p=0.428)$. Comparison

Table I. Demographics

\begin{tabular}{|c|c|c|c|}
\hline Variable & $\operatorname{TSE}(n=30)$ & $\operatorname{TASE}(n=37)$ & $P$-value \\
\hline Male/female & $19 / 11$ & $20 / 17$ & 0.964 \\
\hline Age [years] & $58.67 \pm 8.45$ & $59.70 \pm 10.88$ & 0.671 \\
\hline $\mathrm{BMI}\left[\mathrm{kg} / \mathrm{m}^{2}\right]$ & $22.65 \pm 3.30$ & $22.36 \pm 3.91$ & 0.744 \\
\hline ASA classification: & & & 0.517 \\
\hline 1 & 12 & 18 & \\
\hline$\|$ & 13 & 11 & \\
\hline III & 5 & 8 & \\
\hline IV & 0 & 0 & \\
\hline V & 0 & 0 & \\
\hline Diameter $[\mathrm{cm}]$ & $3.41 \pm 1.40$ & $4.24 \pm 1.95$ & 0.055 \\
\hline
\end{tabular}

$B M I-$ body mass index

Table II. Intraoperative variables

\begin{tabular}{|lccc|}
\hline Variable & TSE $(n=30)$ & TASE $(n=37)$ & $P$-value \\
\hline Operative time $[\mathrm{min}]^{*}$ & $188.27 \pm 70.89$ & $196.43 \pm 80.86$ & $0.559 \mathrm{NS}^{\mathrm{a}}$ \\
\hline Conversion rate & 0 & $5.4 \pm 0.89$ & - \\
\hline Incision length $[\mathrm{cm}]$ & - & $81.76 \pm 70.97$ & 0.687 \\
\hline Blood loss $[\mathrm{ml}]$ & $88.00 \pm 50.61$ & 0 \\
\hline
\end{tabular}

${ }^{*} p \leq 0.05$ represents significant difference. 
of positive lymph nodes in the TSE group and the TASE group was done $(0.10 \pm 0.40$ vs. $1.027 \pm 3.20$; $p=0.089$ ). Circumferential resection margin (CRM) and macroscopic complete total mesorectal excision (MCTME) had no significant difference between the two groups. TME's completion type is classified as complete, almost complete or incomplete [9]. Furthermore, comparison of other pathology results, including resection (R) classification, tumor (T) stage, lymph node (N) stage and TNM stage, was done (Table III).
Postoperative outcomes are presented in Table IV. One patient in the TSE group required blood transfusion, whereas 3 patients in the TASE group required transfusion, and there was no statistical difference ( $p=0.748)$. Comparison of postoperative complications, including intestinal obstruction and anastomotic leak, was done between the two groups. Three patients in the TSE group and 4 patients in the TASE group were confirmed to have anastomotic leakage by stool leaking from a drain. One patient was treated by

Table III. Pathological outcomes

\begin{tabular}{|c|c|c|c|}
\hline Variable & TSE $(n=30)$ & TASE $(n=37)$ & $P$-value \\
\hline Lymph node extraction & $14.23 \pm 7.82$ & $16.03 \pm 10.10$ & 0.428 \\
\hline Positive lymph node & $0.10 \pm 0.40$ & $1.027 \pm 3.20$ & 0.089 \\
\hline R stage: & & & 1.000 \\
\hline RO & 30 & 37 & \\
\hline R1 & 0 & 0 & \\
\hline T stage: & & & 0.128 \\
\hline $\mathrm{T} 1$ & 4 & 1 & \\
\hline $\mathrm{T} 2$ & 8 & 13 & \\
\hline T3 & 25 & 16 & \\
\hline T4 & 0 & 0 & \\
\hline N stage: & & & 0.432 \\
\hline NO & 23 & 25 & \\
\hline N1 & 7 & 12 & \\
\hline N2 & 0 & 0 & \\
\hline TNM stage: & & & 0.623 \\
\hline 1 & 13 & 9 & \\
\hline$\| A$ & 7 & 11 & \\
\hline$\| \mathrm{B}$ & 3 & 5 & \\
\hline IIIA & 2 & 2 & \\
\hline$\| \mathrm{IIB}$ & 3 & 8 & \\
\hline IIIC & 2 & 2 & \\
\hline IV & 0 & 0 & \\
\hline MCTME: & & & 0.082 \\
\hline Complete & 25 & 36 & \\
\hline Nearly-complete & 5 & 1 & \\
\hline Incomplete & 0 & 0 & \\
\hline
\end{tabular}


Table IV. Postoperative outcomes

\begin{tabular}{|c|c|c|c|}
\hline Variable & TSE $(n=30)$ & TASE $(n=37)$ & $P$-value \\
\hline Bowel movement [d] & $3.17 \pm 1.90$ & $3.13 \pm 1.23$ & 0.935 \\
\hline Blood transfusion & 1 & 3 & 0.748 \\
\hline \multicolumn{4}{|c|}{ Postoperative complications: } \\
\hline Anastomotic leak & 3 & 4 & 1.000 \\
\hline Intestinal obstruction & 3 & 1 & 0.318 \\
\hline Length of stay [days] & $10.73 \pm 5.08$ & $10.70 \pm 7.51$ & 0.724 \\
\hline Pain score (day 1) & 3.8 & 5.9 & 0.001 \\
\hline Pain score (day 3) & 2.4 & 3.7 & 0.010 \\
\hline
\end{tabular}

conservative management through drainage placed in the pelvis and the other 6 patients underwent transverse colostomy. All patients were discharged smoothly. Length of stay in the TSE group and the TASE group was similar between the two groups $(10.73 \pm 5.08$ days vs. $10.70 \pm 7.51$ days, $p=0.724$ ). Postoperative pain on the $1^{\text {st }}$ and $3^{\text {rd }}$ postoperative days in the TSE group was significantly less than in the TASE group: the mean postoperative pain scores were 3.8 vs. 5.9 ( $p=0.001)$ and 2.4 vs. $3.7(p=0.010)$ (Table IV).

\section{Discussion}

Some people think that laparoscopic surgery has no advantage over traditional surgery in rectal cancer surgery [8]. With the development of surgical techniques, the surgical procedure of rectal cancer had more choices, such as the conventional open surgery, the laparoscopic technique and the hand-assisted laparoscopic surgery [9]. Oncologic adequacy of the TME specimen was a key determinant in assessing the safety of laparoscopic rectal cancer surgery. Recently, large, prospective, multicenter randomized trials comparing oncologic and long-term outcomes of laparoscopic TSE, TASE and TME have been rare. All TSE cases underwent standard TME surgery, and the mesorectum was without defects and damaged specimens were intact. The reason why we choose a half circle and $10 \mathrm{~cm}$ is the boundary of the operation method. It is mainly considered that if the tumor is too large and the position is high, it is difficult to remove it, but there are also individual differences, which are also related to factors such as the patient's pelvis.

In our study, we found that laparoscopic TSE for radical rectal cancer surgery was safe and effective.
Patients who underwent laparoscopic TSE obtained better cosmetic results, less incision infection and equivalent recovery of bowel function. In addition, the laparoscopic TSE approach for rectal cancer had equivalent MCTME and R classification, CRM, and lymph node retrieval without increasing complication rates when compared with TASE. In our study, the operative time was longer in the TSE group. Many factors might effect the operative time, such as complexity of surgery, experience of surgeon and patient's BMI, etc. The longer time for the procedure of transanal specimen extraction might be another reason.

The TASE also had disadvantages such as the requirement of a 5-7 cm incision for specimen removal. Typically a larger incision carries a greater risk of incision rupture, adhesions, and incision hernia [10-14]. In this study the incision infection rate of the TASE group was $10.81 \%$ and that of the TSE group was zero. It has been reported that in the surgical group undergoing TASE, the incidence of postoperative incision infection and the incidence of incisional hernia are $5-11.1 \%$ and $1-7.7 \%$ [15-20], respectively. The reason for the high infection rate of the incision is as follows: the adherence of the sterility principle when changing the dressing is not strict; for the sake of rigor, fat liquefaction is also classified as an infection.

Our current experience shows that TSE combined with TME and laparoscopic rectal cancer technology is suitable for radical tumor resection and is a minimally invasive procedure. The rectal stump is a "necessary" trauma, through which we could precisely determine the distal cutting edge of the rectum under direct vision. The blood supply to the descending colon could also be determined easily. 
In the present study, we also found that overall postoperative complications were similar between the two groups. As everyone knows, postoperative complications are affected by many factors, such as modes of operation, experience of the surgeon and physical condition of patients before the operation. All patients had no postoperative incontinence, and the causes were as follows: 1 . The location of the tumor was mainly in the middle and high position; 2. The neurological function was intact under the endoscope; 3. The transient bowel habit was changed, and it returned to normal within 3 months to 6 months.

In terms of pathological outcomes, there was no significant difference between the two groups in our study. The present study showed similar lymph node extraction, positive lymph node and $\mathrm{R}$ classification. CRM and MCTME were important factors for the operative quality assessment and prognosis. Based on the specimen examination, we found that there was no significant difference of CRM and MCTME between the two groups. The College of American Pathologists (CAP) recommended examination of a minimum of 12 lymph nodes to declare that a patient has node-negative disease. Although the lymph node number in the TSE group was slightly less compared to the TASE surgery group in our study, the number was still more than 12 for both groups. There is no special difference in the postoperative data between TSE and TASE. The advantage should be in cosmesis and no incision. However, there should be a clearer guide on how to choose indications and how to make choices during preoperative surgery.

This study had several limitations that deserve attention. It was retrospective, single-center study, and a single surgeon performed all of the procedures. Selection of patients for one approach versus the other was subject to selection biases, because the patients were selected according to surgeon preference and other factors, such as patient characteristics, disease complexity and operative factors. In addition, the relatively small number of patients was another drawback of our study. Moreover, surgical cost, postoperative pain scores and narcotic use were not assessed. Although we did not include an assessment of some meaningful parameters, we did evaluate operative time, blood transfusion, postoperative complications, pathological outcomes, length of stay, and so on. Finally, a long-term follow-up and a large-scale randomized controlled trial in direct comparison of TSE and TASE are required to assess more definitely the standard oncologic outcomes such as survival and recurrence. Because TME needs lower anastomosis, anastomotic leakage is easy to occur after TME. Norgren et al. found that the incidence of anastomotic leakage in 219 cases of TME after operation was $11 \%$. Carlsen reported that the incidence of anastomotic leakage after TME was $11-16 \%$, which was higher than $8 \%$ in the non-TME group. It was easy to occur in patients with low anastomosis and long operation time. Improving surgical skills and shortening operation time were the key to solving the problem. Temporary prophylactic colostomy can prevent anastomotic leakage. TME requires fine anatomy during the operation, so it is bound to increase the operation time and may cause excessive bleeding. By mastering the operation skills of TME skillfully, it is possible to shorten the operation time and avoid postoperative anastomotic leakage.

\section{Conclusions}

Short-term outcomes after TSE for laparoscopic rectal cancer surgery are similar to those after TASE, with equivalent recovery of bowl, no statistically significant difference in operative time, shorter incision length, and lower postoperative pain index. TSE is an ideal approach for the treatment of rectal cancer.

\section{Acknowledgments}

This research was supported by Medical Scientific Research Foundation of Guangdong Province, China (No. A2016055).

\section{Conflict of interest}

The authors declare no conflict of interest.

\section{References}

1. Heald RJ, Ryall RD. Recurrence and survival after total mesorectal excision for rectal cancer. Lancet 1986; 1: 1479-82.

2. Swaid F, Sroka G, Madi H, et al. Totally laparoscopic versus laparoscopic-assisted left colectomy for cancer: a retrospective review. Surgical Endoscopy 2016; 30: 2481-8.

3. Guillou PJ, Quirke P, Thorpe H, et al.; MRC CLASICC trial group. Short-term endpoints of conventional versus laparoscopic-assisted surgery in patients with colorectal cancer (MRC CLASI CC trial): multicentre, randomised controlled trial. Lancet 2005; 365: 1718-26.

4. McLeod RS, Stern H. Laparoscopy-assisted colectomy versus open colectomy for treatment of nonmetastatic colon cancer: a randomized trial. Can J Surg 2004; 47: 209-11. 
5. Małczak P, Mizera M, Torbicz G, et al. Is the laparoscopic approach for rectal cancer superior to open surgery? A systematic review and meta-analysis on short-term surgical outcomes. Videosurgery Miniinv 2018; 13: 129-40.

6. Bae SU, Jeong WK, Baek SK, et al. Reduced-port robotic total mesorectal resection for rectal cancer using a single-port access: a technical note. Videosurgery Miniinv 2017; 12: 378-84.

7. Han F, Hua L, Zhao Z, et al. Transanal natural orfice specimen extraction for laparoscopic rectal cancer anterior resection. World J Gastroenterol 2013; 19: 7751-7.

8. Pędziwiatr M, Małczak P, Mizera M, et al. There is no difference in outcome between laparoscopic and open surgery for rectal cancer: a systematic review and meta-analysis on short- and long-term oncologic outcomes. Tech Coloproctol 2017; 21: 595-604.

9. Zawadzki M, Krzystek-Korpacka M, Gamian A, Witkiewicz W. Serum cytokines in early prediction of anastomotic leakage following low anterior resection. Videosurgery Miniinv 2018; 13: 33-43.

10. Nagtegaal ID, van de Velde CJ, van der Worp E, et al. Cooperative clinical investigators of the Dutch Colorectal Cancer Group. Macroscopic evaluation of rectal cancer resection specimen: clinical significance of the pathologist in quality control. J Clin Oncol 2002; 20: 1729-34.

11. Targarona EM, Gracia E, Garriga J, et al. Prospective randomized trial comparing conventional laparoscopic colectomy with hand-assisted laparoscopic colectomy: applicability, immediate clinical outcome, inflammatory response, and cost. Surg Endosc 2002; 16: 234-9.

12. Ozturk E, Kiran RP, Remzi F, Geisler D, Fazio V. Hand-assisted laparoscopic surgery may be a useful tool for surgeons early in the learning curve performing total abdominal colectomy. Colorectal Dis 2010; 12: 199-205.

13. DeSouza A, Domajnko B, Park J, et al. Incisional hernia, midline versus low transverse incision: what is the ideal incision for specimen extraction and hand-assisted laparoscopy? Surg Endosc 2011; 25: 1031-6.

14. Ringley C, Lee YK, Iqbal A, et al. Comparison of conventional laparoscopic and hand-assisted oncologic segmental colonic resection. Surg Endosc 2007; 21: 2137-41.

15. Larson DW, Cima RR, Dozois EJ, et al. Safety, feasibility, and short-term outcomes of laparoscopic ileal-pouch-anal anastomosis: a single institutional case-matched experience. Ann Surg 2006; 243: 667-70.

16. Fabozzi M, Allieta R, Contul RB, et al. Comparison of short- and medium-term results between laparoscopically assisted and totally laparoscopic right hemicolectomy: a case-control study. Surg Endosc 2010; 24: 2085-91.

17. Tan WS, Chew MH, Ooi BS, et al. Laparoscopic versus open right hemicolectomy: a comparison of short-term outcomes. Int J Colorectal Dis 2009; 24: 1333-9.

18. Zheng MH, Feng B, Lu AG, et al. Laparoscopic versus open right hemicolectomy with curative intent for colon carcinoma. World J Gastroenterol 2005; 11: 323-6.

19. Dusek T, Orhalmi J, Sotona O, et al. Neopterin, kynurenine and tryptophan as new biomarkers for early detection of recta anastomotic leakage. Videosurgery Miniinv 2018; 13: 44-52.
20. Winslow ER, Fleshman JW, Birnbaum EH, Brunt LM. Wound complications of laparoscopic vs. open colectomy. Surg Endosc 2002; 16: 1420-5.

Received: 3.09.2018, accepted: 8.10.2018. 